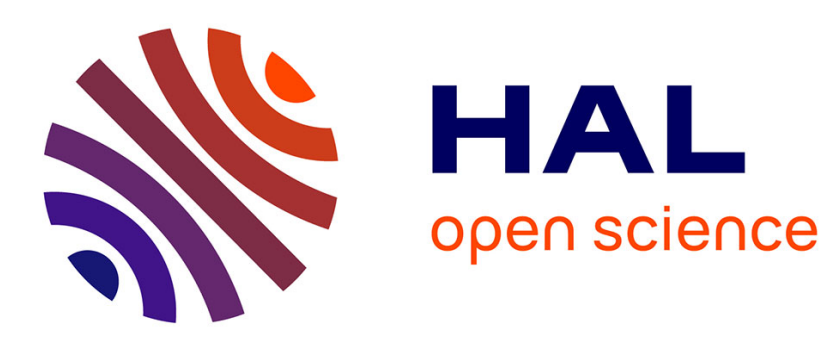

\title{
Loi de comportement ARMOR pour géomatériaux sous chargement dynamique
}

\author{
C. Mariotti, F. Thomas
}

\section{To cite this version:}

C. Mariotti, F. Thomas. Loi de comportement ARMOR pour géomatériaux sous chargement dynamique. Journal de Physique IV Proceedings, 1994, 04 (C8), pp.C8-577-C8-582. 10.1051/jp4:1994890 . jpa-00253451

HAL Id: jpa-00253451

https://hal.science/jpa-00253451

Submitted on 1 Jan 1994

HAL is a multi-disciplinary open access archive for the deposit and dissemination of scientific research documents, whether they are published or not. The documents may come from teaching and research institutions in France or abroad, or from public or private research centers.
L'archive ouverte pluridisciplinaire HAL, est destinée au dépôt et à la diffusion de documents scientifiques de niveau recherche, publiés ou non, émanant des établissements d'enseignement et de recherche français ou étrangers, des laboratoires publics ou privés. 


\section{Loi de comportement ARMOR pour géomatériaux sous chargement dynamique}

C. Mariotti et F. Thomas

Commissariat à l'Energie Atomique, Direction des Applications Militaires, Laboratoire de Géophysique, BP. 12, 91680 Bruyères-le-Châtel, France

Résumé : Un modèle de comportement dynamique des géomatériaux qui traite la fracturation par une variable d'endommagement est décrit. Ce modèle exploite au maximum les données expérimentales et en particulier les courbes de détente mesurées lors d'impacts plans. Le mécanisme isotrope et le mécanisme déviatorique sont couplés. Une simulation numérique d'explosion sphérique divergente dans un Quartzite illustre les possibilités du modèle.

Abstract : A constitutive model for geomaterial is described. This model uses all the experimental data and in particular the paths of the isotropic mechanism. The fracturation is described by a damage variable. The isotropic mechanism and the deviatoric mechanism are coupled. Some experimental spherical divergent explosions on a Quartzite Rock are simulated.

\section{INTRODUCTION.}

Les études numériques des phénomènes de dynamique rapide dans les géomatériaux nécessitent l'élaboration de lois de comportement adaptées. Dans ce but, nous avons développé un modèle nommé ARMOR (A Rheological MOdel for Rocks) qui dans son formalisme, exploite au maximum les données expérimentales. Ce modèle lagrangien comporte deux mécanismes isotrope et déviatoire couplés dans le domaine fragile. La fracturation est décrite par une variable d'endommagement scalaire $\mathrm{D}$ qui fait évoluer l'enveloppe de résistance des matériaux. Ce modèle s'inspire des lois SOC et TENSOR [1].

\section{LA PHYSIQUE DE LA LOI ARMOR.}

\subsection{Modélisation du mécanisme isotrope}

Le mécanisme volumique est représenté par une relation entre la pression $p$ et une représentation $\mu$ de la variation de volume :

avec

$$
\mathrm{p}=\mathrm{p}(\mu)
$$

$$
\begin{aligned}
& p=1 / 3 \operatorname{tr}(\sigma) \\
& \mu=\rho / \rho o-1
\end{aligned}
$$

où $\rho$ et $\rho$ o sont respectivement la masse volumique et la masse volumique initiale et $\sigma$ les contraintes. 


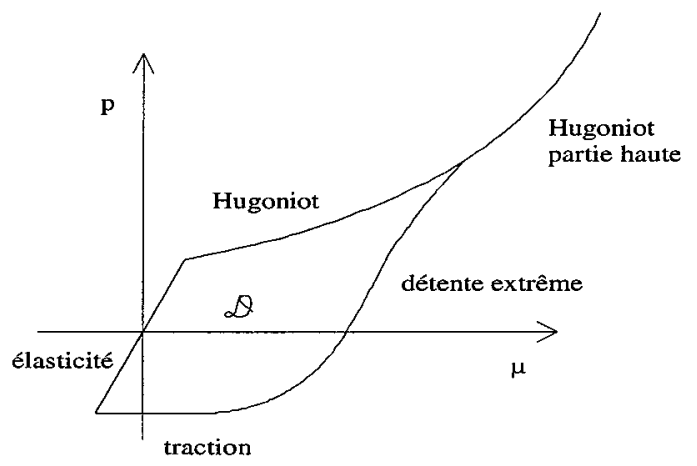

Fig.1.Domaine du mécanisme isotrope
Le mécanisme volumique est défini à l'intérieur d'un domaine $D$ délimité par quatre courbes : l'Hugoniot, la limite de traction, la détente extrême et la courbe élastique ; et par une courbe dans le domaine des pressions plus élevées qui est la suite de l'Hugoniot (cette courbe sera désignée par la suite comme la partie haute de l'Hugoniot) (Fig.1).

En fonction du chargement, le point de charge évolue sur une courbe de détente, sur l'Hugoniot ou sur la limite de traction. La connaissance de $\mathrm{d} \mu$ suffit à la détermination de $\mathrm{dp}$. Mais il est nécessaire pour cela de connaître l'ensemble des courbes de détente du domaine. Ceci peut se faire par une méthode d'interpolation entre des courbes de détente déterminées expérimentalement. Nous allons décrire la méthode numérique retenue pour faire cette interpolation.

Sur le plan numérique, on réalise un maillage du domaine $\mathcal{D}$ s'appuyant sur les courbes de détente intermédiaires quand elles existent. Les noeuds de ce maillage sont donc issus des données expérimentales relatives aux états de détente. Ceci permet d'utiliser au mieux l'ensemble des données des courbes de détente disponibles. Un maillage régulier de référence est également défini dans un système de coordonnées curvilignes (s,t) (Fig.2).

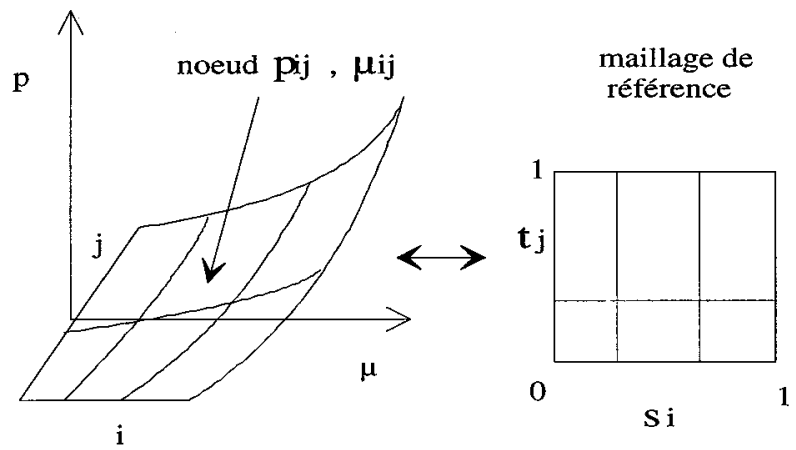

Fig.2. Maillage pour l'interpolation du mécanisme isotrope

Une courbe de détente correspond ainsi à une droite dans le domaine de référence :

$$
\mathrm{s}=\text { constante }
$$

Afin de conserver des continuités au niveau des dérivées premières et secondes, les fonctions de base d'interpolation de ce domaine de référence sont des fonctions bicubiques des abscisses curvilignes . Les variables physiques p et $\mu$ sont alors discrétisées sur le domaine de référence par rapport aux coordonnées curvilignes grâce à ces fonctions de base. La pression $\mathrm{p}$ et $\mu$ sont interpolés par deux fonctions bicubiques indépendantes :

$$
\begin{aligned}
& p=f(s, t) \\
& \mu=g(s, t)
\end{aligned}
$$


où $\mathrm{s}$ et $\mathrm{t}$ sont les coordonnées curvilignes d'un maillage réglé régulier de référence

$$
\begin{aligned}
& 0<\mathrm{s}<1 \\
& 0<\mathrm{t}<1
\end{aligned}
$$

A partir de ce maillage régulier, la définition mathématique de ces fonctions $\mathrm{f}$ et $\mathrm{g}$ offre la possibilité de calculer la célérité des ondes par la dérivation des courbes de détente.

\subsection{Modélisation du mécanisme déviatoire}

Le mécanisme déviatoire est défini dans le plan $(\mathrm{p}, \mathrm{q})$ où $q$ est la grandeur définie par :

$$
q^{2}=3 / 2 s_{i j}^{2}=3 J_{2}
$$

avec $s_{i j}$ partie déviatorique des composantes du tenseur des contraintes et $J_{2}$ second invariant du déviateur.

Pour ce mécanisme déviatoire, un domaine des faibles pressions, dit fragile, est défini pour p inférieure à une pression de transition ( $\mathrm{Pdf}$ ) entre le domaine dit fragile et le domaine dit ductile (pression $\mathrm{p}$ supérieure à Pdf). Dans le domaine fragile, plusieurs courbes intrinsèques sont définies en fonction d'une variable d'endommagement traduisant l'état de fracturation du milieu. Dans le domaine ductile, ces courbes intrinsèques se rejoignent pour n'en former plus qu'une seule. La courbe intrinsèque désigne l'ensemble des limites de rupture pour les divers essais réalisés et correspond au seuil de rupture (Fig.3). La courbe seuil $S$ du matériau est définie par une interpolation entre le seuil du matériau sain $S_{S}$ et celui du matériau fracturé $S_{\mathrm{f}}$ à l'aide de la variable d'endommagement scalaire D

$$
\begin{aligned}
& S=S_{S}(1-D)+S_{f} D \\
& 0<D<1
\end{aligned}
$$

La partie déviatorique des composantes du tenseur des contraintes, sij, est obtenue par la relation élastoplastique :

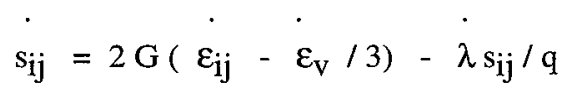

où $\varepsilon_{\mathrm{ij}}$ et $\varepsilon_{\mathrm{v}}$ sont le tenseur des déformations et la déformation volumique. (le point désigne la dérivée par rapport au temps ). G est le module de cisaillement.

Le terme en $\lambda$ positif ou nul permet de projeter le tenseur des contraintes sur la courbe intrinsèque dans le cas de déformations plastiques ( cas où $q=S(p)$ ).

La courbe intrinsèque $S$ évolue également en fonction de l'endommagement $D$ (Fig.3).

Si le point de charge se situe dans le domaine fragile plastique :

$$
q>S(p) \text { et } p<P d f
$$

alors il y a évolution de l'endommagement suivant une fonction de la forme :

$$
\mathrm{dD}=\mathrm{dD}(\mathrm{L}, \mathrm{dL})
$$

où $\mathrm{L}$ est la longueur de fracturation et $\mathrm{dL}$ son accroissement. 


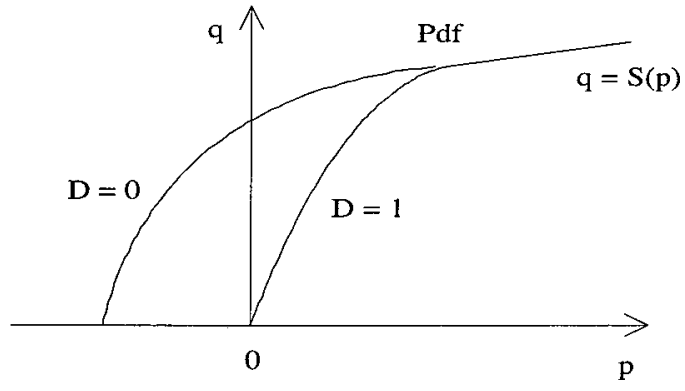

Fig.3. Domaine du mécanisme déviatoire

Si le point de charge se situe dans le domaine ductile plastique :

$$
q>S(p) \text { et } p>P d f
$$

alors l'endommagement est immédiatement passé à 1 car on suppose que le matériau est complètement endommagé.

Si le point de charge est dans le domaine élastique, il n'y a pas d'évolution de l'endommagement.

$$
\mathrm{dD}=0
$$

Il reste à déterminer la fonction d'évolution de l'endommagement correspondant aux mécanismes de rupture recherchés.

\subsection{Evolution de l'endommagement}

Pour décrire l'évolution du paramètre d'endommagement $\mathrm{D}$, il faut se donner une loi de distribution des défauts initiaux du milieu et un mécanisme de croissance de ces défauts. La distribution retenue est celle de Mott :

$$
F_{\text {cum }}(s)=\exp \left(-\left(2 s / s^{*}\right)^{1 / 2}\right)
$$

qui correspond à la fraction cumulée des fragments ayant une surface supérieure à s, s* étant une surface moyenne des fragments [2]. La présence de la racine carrée dans cette distribution montre que la variable physique caractéristique du phénomène est une longueur.

Pour décrire le matériau, une distribution des fractures de longueur $\mathrm{L}$ de la forme classique suivante est choisie :

$$
\mathrm{N}(\mathrm{L})=\exp (-\mathrm{L} / \mathrm{Lo})
$$

Lo étant la longueur moyenne des fractures de cette distribution. Cette longueur Lo est caractéristique de l'état de fracturation final du matériau. Elle fait partie des données fournies par l'utilisateur. Lo a une dimension fractale [3] attachée à la taille globale du domaine. Ainsi, la valeur prise par Lo pour un calcul sur un bloc de $1 \mathrm{~m}$ pourra être plus petite que celle prise pour un bloc de $1 \mathrm{~km}$.

Pour la distribution de fractures choisie, si à un instant donné, la fracturation a une taille moyenne $L$ et si on considère qu'elle se développe selon des formes planes circulaires (Penny Shape), l'accroissement de la surface fracturée, c'est à dire l'endommagement, correspond au produit du nombre de fractures par l'accroissement de la surface des fractures. Ainsi D est une fonction de

$$
\exp (-\mathrm{L} / \mathrm{Lo}) \mathrm{L} \mathrm{dl} /(\mathrm{Lo} * \mathrm{Lo})
$$

Il est classique [4] de prendre pour décrire l'évolution de l'endommagement la forme suivante :

$$
(1-\mathrm{m})(1-\mathrm{D})^{-\mathrm{m}} \mathrm{dD}=\exp (-\mathrm{L} / \mathrm{Lo}) \mathrm{L} \mathrm{d} \mathrm{l} / \mathrm{Lo}^{2}
$$

où $\mathrm{m}$ est un paramètre compris entre 0 et 1 qui permet de traduire la non linéarité de la vitesse d'endommagement en fonction de l'endommagement. 
L'incrément de la longueur de fissure reste déterminé à partir des célérités de propagation de fissure en traction $\mathrm{Ct}$ ou en compression $\mathrm{Cc}$ qui sont deux variables physiques données dans le jeu de paramètres :

$$
\mathrm{dl}=\mathrm{Cdt}
$$

où $\mathrm{C}$ est égal à $\mathrm{Ct}$ ou $\mathrm{Cc}$ suivant que la contrainte principale mineure est en traction ou en compression

\subsection{Couplage entre les deux mécanismes}

Les deux mécanismes sont définis par des surfaces de charges. La surface de charge du mécanisme déviatorique évolue en fonction d'une variable d'endommagement. Dans la conception du modèle, ces deux surfaces de charge sont les projections dans deux plans particuliers d'une seule et unique surface de charge dans l'espace $(p, q, \mu)[5]$. De ce fait, il faut modifier la surface de charge du mécanisme isotrope quand la surface de charge du mécanisme déviatorique bouge.

En particulier, la limite à la traction qui correspond à une droite $\mathrm{AB}$ pour le mécanisme isotrope et à un point $\mathrm{L}$ pour le mécanisme déviatorique doivent toujours être en concordance (Fig.4). Au cours de l'endommagement le point L se déplace vers l'origine des pressions. Afin de maintenir cette concordance entre la droite $\mathrm{AB}$ et le point $\mathrm{L}$, on applique une fonction mathématique de type homothétique sur la fonction de charge du mécanisme isotrope ; 1 est alors aligné avec ab. Cette fonction va réduire selon l'axe des pressions la fonction de charge isotrope. Il faut au moins utiliser une transformation parabolique dans cette réduction afin de conserver des continuités dans les dérivations.

Le segment $\mathrm{AB}$ converge donc vers l'axe des abscisses $(p=0)$ au fur et à mesure de l'augmentation de $\mathrm{D}$, le domaine $D$ évolue avec D. Cette transformation mathématique parabolique conduit à réduire la pente des courbes de détentes au fur et à mesure de l'endommagement ce qui signifie une diminution de la célérité des ondes avec l'endommagement.

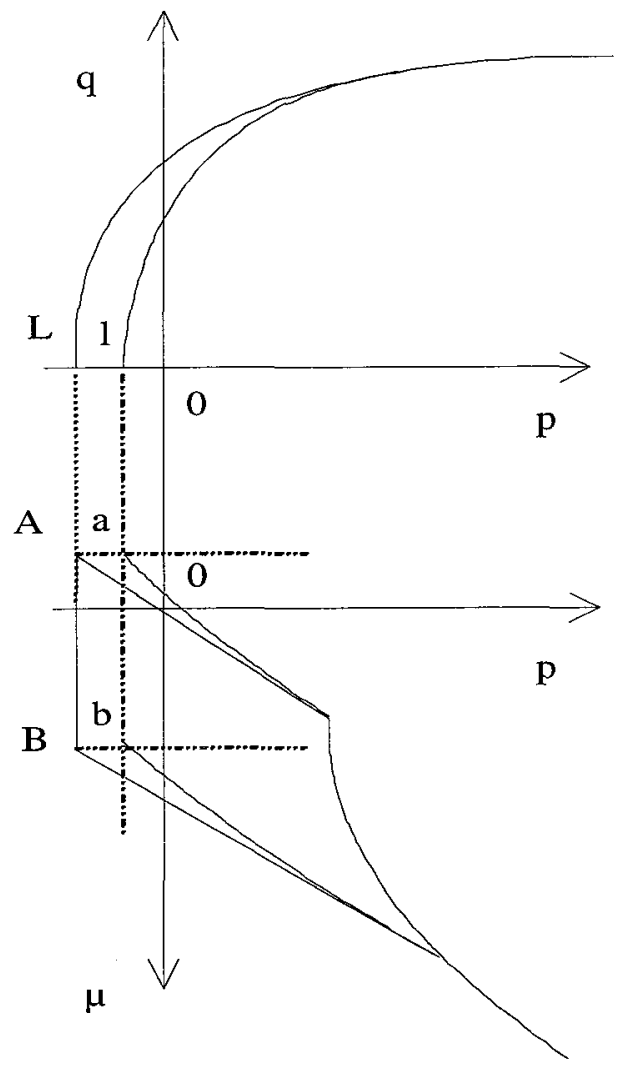

Fig.4. Couplage entre les mécanismes

\section{COMPARAISON AVEC DES RESULTATS EXPERIMENTAUX.}

Des essais d'explosion sphérique divergente ont été réalisés au Centre d'Etude de Gramat sur une roche de Quartzite [6]. L'essai consiste à faire exploser une charge hémisphérique de rayon $1,83 \mathrm{~cm}$ à la surface d'un cylindre de Quartzite de rayon $8 \mathrm{~cm}$ et de hauteur $20 \mathrm{~cm}$ confiné par du béton de même impédance . Après essai, le bloc de Quartzite est récupéré et permet une comparaison qualitative de la fracturation avec l'endommagement déterminé par une simulation numérique conduite a priori. La partie supérieure du Quartzite proche de l'explosif a été pulvérisée et correspond parfaitement à la zone d'endommagement 
maximal $(\mathrm{D}=1)$. Sur le côté de l'échantillon des écailles se sont détachées faisant apparaître une forme de pain de sucre. Cette forme se retrouve au niveau de la carte d'endommagement calculée (Fig. 5), les cadres de la figure représentent la taille initiale du bloc de Quartzite. La comparaison entre l'expérience et des calculs menés a priori semble très satisfaisante.

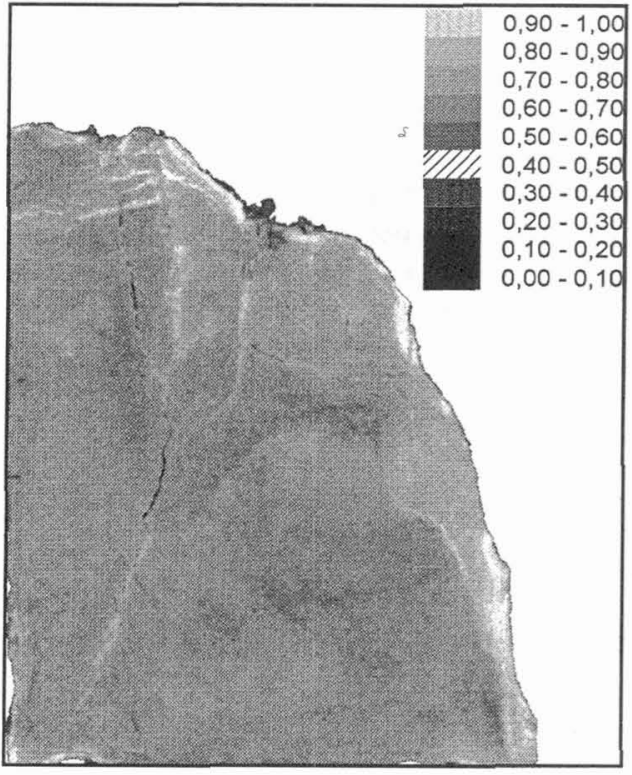

Fig.5. Coupe longitudinale du bloc de Quartzite après essai

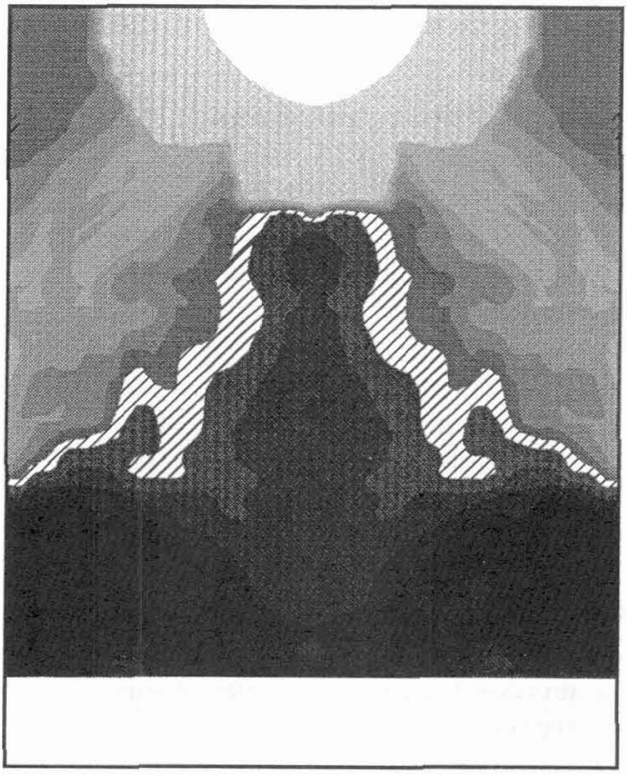

Carte de l'endommagement d'après la simulation numérique

\section{CONCLUSION.}

La loi ARMOR répond aux objectifs fixés dans le cadre de la création d'une loi pragmatique pour l'exploitation de calculs dynamiques. Ce modèle se veut proche de la réalité expérimentale et les paramètres sont directement reliés à la physique. Sa confrontation à des expériences s'avère prometteuse.

\section{REFERENCES}

[1] Schatz J.F., "The physics of SOC and TENSOR" Lawrence Livermore Laboratory, Rept UCRL $51352,1973$.

[2] Englman R., Jaeger Z., Levi A., "Percolation theoretical treatment of two-dimensional fragmentation in solids" Philosophical Magazine B, 1984, Vol. 50, $\mathrm{n}^{\circ} 2,307-315$.

[3] Fractals in Physics, Proceedings of the Sixth International Symposiuml on Fractals in Physics, International Centre for Theoretical Physics, Trieste, Italy , 1985, edited by L. Pietronero and E. Tosatti (North-Holland, Amsterdam, 1986)

[4] Lemaitre J., Chaboche J.L, Mécanique des matériaux solides, 1984, DUNOD.

[5] Critescu N., Rock Rheology, 1989, Published by Kluwer Academic Publishers.

[6] Tranchet J.Y., "Comportement de deux matériaux polycristallins sous l'effet de la propagation d'une onde sphérique divergente", Thèse à paraître à l'Université de Bordeaux I, 1994. 